\title{
1 Improving maps of forest aboveground biomass: A 2 combined approach using machine learning with a $3 \quad$ spatial statistical model
}

\author{
4 Shaoqing Dai ${ }^{1,2, *}$, Xiaoman Zheng ${ }^{1,2, *}$, Lei Gao ${ }^{3}$, Chengdong Xu ${ }^{4}$, Shudi Zuo ${ }^{1,2,5}$, Qi \\ 5 Chen $^{6}$, Xiaohua $\mathrm{Wei}^{7}$, Yin Ren ${ }^{1,5}$ \\ ${ }^{1}$ Key Laboratory of Urban Environment and Health, Key Laboratory of Urban Metabolism of Xiamen, \\ 8 Institute of Urban Environment, Chinese Academy of Sciences, CN 361021, China \\ $9 \quad{ }^{2}$ University of Chinese Academy of Sciences, CN 100049, China \\ $10 \quad{ }^{3}$ CSIRO, Waite Campus, Urrbrae, SA 5064, Australia \\ $11{ }^{4}$ State Key Laboratory of Resources and Environmental Information System, Institute of Geographic \\ 12 Sciences and Natural Resources Research, Chinese Academy of Sciences, CN 100049, China \\ 5 Ningbo Urban Environment Observation and Research Station-NUEORS, Chinese Academy of \\ Sciences, CN 315800, China \\ ${ }^{6}$ Department of Geography, University of Hawai'i at Mānoa, Honolulu, HI 96822, USA \\ ${ }^{7}$ Department of Earth and Environmental Sciences, University of British Columbia, Kelowna, BC V1V \\ 1V7, Canada \\ *These authors contributed equally to this work.
}


https://doi.org/10.5194/bg-2020-36

Preprint. Discussion started: 25 February 2020

(c) Author(s) 2020. CC BY 4.0 License.

22 Abstract: Aboveground biomass (AGB) estimates at the plot level plays a major part in connecting accurate single-tree AGB measurements to relatively difficult regional-scale AGB estimates. However, complex and spatially heterogeneous landscapes, where multiple environmental covariates (such as longitude, latitude, and forest structure) affect the spatial distribution of AGB, make upscaling of plot-level models more challenging. To address this challenge, this study proposes an approach that combines machine learning with spatial statistics to construct a more accurate plot-level AGB model. The study was conducted in a Eucalyptus plantation in Nanjing, China. We developed, evaluated, and compared the accuracy and performance of three different machine learning models [support vector machine (SVM), random forest (RF), and the radial basis function artificial neural network (RBF-ANN)], one spatial statistics model (P-BSHADE), and three combinations thereof (SVM \& P-BSHADE, RF \& P-BSHADE, RBF-ANN \& P-BSHADE) for forest AGB estimates based on AGB data from 30 sample plots and their corresponding environmental covariates. The results show that the performance indices RMSE, nRMSE, MAE, and MRE of all combined models are substantially smaller than those of any individual models, with the RF \& P-BSHADE combined method giving the smallest value. These results demonstrate clearly that combined models, especially the RF \& P-BSHADE model, can improve the accuracy of plot-level AGB models and reduce uncertainty on plot-level AGB estimates or even on large-forested-landscape AGB estimates. These research results are important because they reduce the uncertainty in estimates of the regional carbon balance. 


\section{Introduction}

Accurate maps of aboveground biomass (AGB) provide a solid foundation for sound decision-making in sustainable forest management scenarios, such as reducing deforestation, forest degradation, and greenhouse-gas emissions (Bustamante et al., 2016; Houghton et al., 2009; Mendoza-Ponce and Galicia, 2010). Most AGB maps are constructed based on plot-level estimation models, which are challenging to scale up and can ultimately propagate uncertainty to regional AGB maps. The uncertainty of such regional maps can be attributed to two primary sources: (1) the use of inadequate sampling data to construct the plot level prediction models, and (2) model-dependent uncertainty, including unreasonable model-parameter assumptions and improper model structure (Chen et al., 2015; Gao et al., 2016; McRoberts et al., 2016). The present study mainly focuses on reducing the second source of uncertainty.

An estimated $18 \%-103 \%$ of the uncertainty in AGB mapping can be attributed to model-dependent uncertainty (Djomo and Chimi, 2017; Malhi et al., 2004). Although the allometric model, which is the most popular plot-level model, has produced useful results for forest AGB estimates (Conti et al., 2019; Huang et al., 2019), selection error in plot-level allometric modeling still leads to over $40 \%$ uncertainty (Djomo et al., 2016; Fayolle et al., 2013; Chave et al., 2014), and simple or complex forms of the allometric model account for $20 \%-60 \%$ of the uncertainty (Picard et al., 2015).

Many different plot-level prediction models other than allometric models have been applied to constructing accurate AGB maps, including linear models (Andersen et al., 2014; Morel et al., 2012), machine learning models (Chen, 2015; Gleason and Im, 2012), and spatial statistical models (Benitez et al., 2016; Propastin, 2012; Van der Laan et al., 2014). With the development of computer-science techniques and advances in nonlinear biomass modeling, machine learning methods have become prevalent. Traditional parametric methods, which summarize data with a fixed number of parameters based on sample size (e.g., logistic regression and perceptron) (Gao and Hailu, 2012), have difficulty characterizing nonlinear relationships between AGB and multiple environmental covariates. By comparison, nonparametric machine learning algorithms, in which the number of parameters depends on the number of training examples (e.g., K-nearest neighbor, support vector machine, and random forest), are advantageous because they are more elastic and do not restrict variable types, the distribution of predictor variables, or the relationship between response and predictor variables (Lu et 
al., 2007). In addition, nonparametric machine learning algorithms may offer higher prediction accuracy

(Frey et al., 2019; Gleason and Im, 2012).

Another group of models frequently used to estimate the relationship between forest AGB and multiple environmental covariates is based on spatial statistical approaches, including geographically weighted regression and Kriging (Du et al., 2010; Van der Laan et al., 2014; Viana et al., 2012). Spatial statistical methods are based on analyses of attribute information, such as spatial location (Schabenberger and Gotway, 2005). Compared with traditional statistical methods, spatial methods integrate spatial factors that affect model responses, thus removing the constraints of traditional statistical methods that assume sample independence (Rangel and Bini, 2010) and improving our understanding of spatial autocorrelation and heterogeneity (He et al., 2011; Rosenberg and Anderson, 2011).

Although many studies have integrated ground-based plot data, multi-source remote-sensing data (e.g., LiDAR and Landsat), and machine learning or spatial statistical methods, the prediction accuracy of current AGB spatial mapping still suffers from uncertainty (McRoberts et al., 2018; Paul et al., 2016; Saatchi et al., 2011; Zheng et al., 2004; Jachowski et al., 2013; Zhang et al., 2014). First, existing studies that used machine learning methods have not considered the spatial heterogeneity of multiple environmental covariates (such as longitude, latitude, and forest structure), which affects the spatial distribution of AGB (Babcock et al., 2015; Fassnacht et al., 2014). Second, the assumptions of the spatial statistical method (e.g., spatial autocorrelation and spatial stratified heterogeneity) may not always apply to forest AGB.

AGB estimates at the plot level serve as a bridge to connect single-tree AGB measurements to AGB estimates on a regional scale. Accurate AGB mapping at the plot scale provides a basis for future upscaling to the regional scale. However, the uncertainty and error propagation inherent in different prediction models make this process challenging. Allometric models are most commonly used to construct plot-level AGB models, but they cannot fully capture the complex and spatially heterogeneous landscapes where multiple environmental covariates (such as longitude, latitude, and forest structure) affect the spatial distribution of AGB. The objective of the present study is to develop and evaluate a combined machine learning and spatial statistical method that uses ground-based samples to improve the prediction accuracy of AGB spatial mapping at the plot level. The proposed method 
https://doi.org/10.5194/bg-2020-36

Preprint. Discussion started: 25 February 2020

(C) Author(s) 2020. CC BY 4.0 License.

integrates the nonlinear mapping capabilities of machine learning algorithms [i.e., radial basis function artificial neural network (RBF-ANN), support vector machine (SVM), and random forest (RF)] with the spatial autocorrelation and stratified heterogeneous advantages of a spatial statistical model (i.e., the point estimation model of biased sentinel hospital-based area disease estimation, P-BSHADE) (Xu et al., 2013). Our aim is to answer two specific questions: (1) What are the differences in prediction accuracy of AGB maps based on different methods? (2) Can the integration of spatial statistical and machine learning methods improve the accuracy of AGB models at the plot level? We explore these two questions by studying an empirical case for predicting an AGB map at a Eucalyptus plantation in Nanjing County, China.

\section{Materials and Methods}

\subsection{Site description}

Nanjing County $\left(117^{\circ} 00^{\prime}-117^{\circ} 36^{\prime} \mathrm{E}, 24^{\circ} 26^{\prime}-25^{\circ} 00^{\prime} \mathrm{N}\right.$, Fig. $\left.1 \mathrm{~b}\right)$ is located in the upstream region of the Jiulong River in Fujian Province, China. Seventy-four percent (145009 ha) of the county comprises forests and 79346 ha are plantations. The region is affected by the South Asian tropical monsoon climate. In 2014, the average annual temperature in Nanjing County was $21.1^{\circ} \mathrm{C}$, with an annual precipitation of $1700 \mathrm{~mm}$ and 340 frost-free days. The major soil type is red soil.

The study area has a complex topography with significantly varying elevation $(0-1566 \mathrm{~m})$. Forest composition, structure, and biomass are spatiotemporally heterogeneous. The main tree species are Eucalyptus grandis $x$ urophylla, Pinus massoniana, and Cunninghamia lanceolata. Recently, the area of Eucalyptus plantations has increased rapidly, reaching 13338 ha, which is an increase of 10862 ha in one decade. 
https://doi.org/10.5194/bg-2020-36

Preprint. Discussion started: 25 February 2020

(c) Author(s) 2020. CC BY 4.0 License.

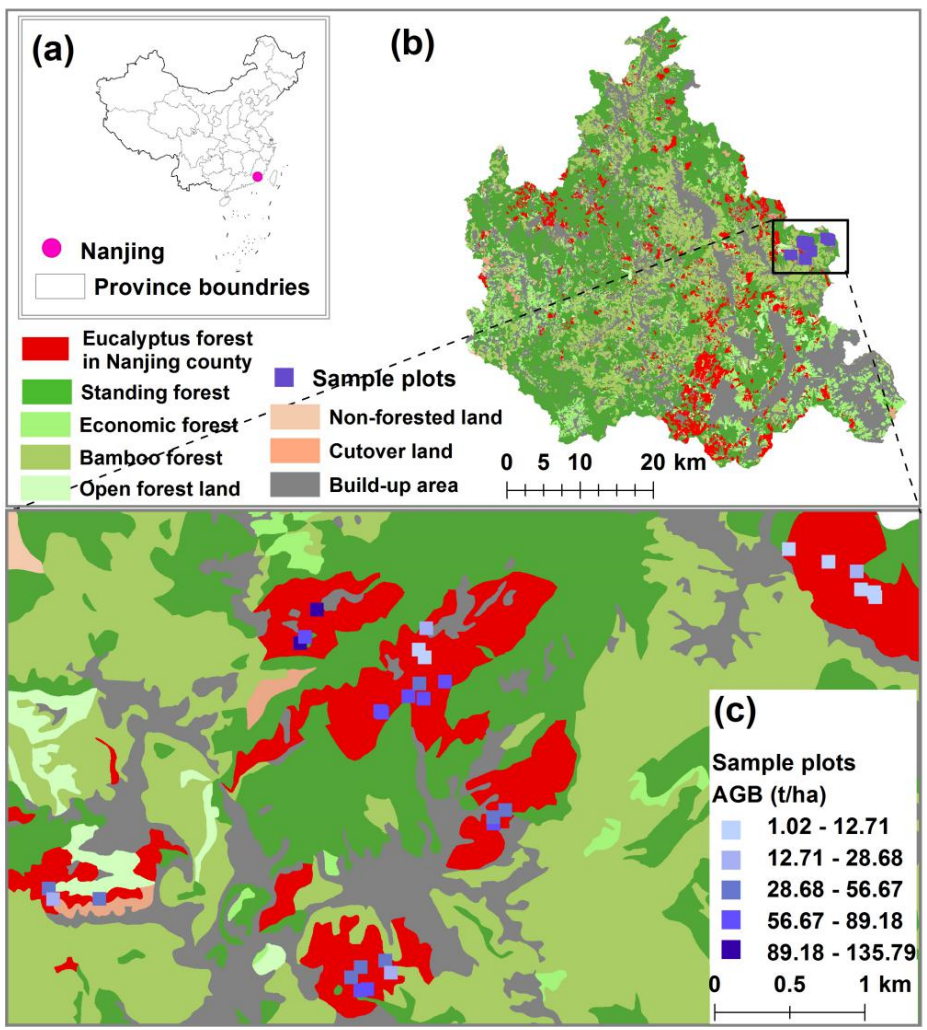

Figure 1. The study area is a typical example of a non-representative-sample problem. (a) other major forests. (c) Spatial distribution of the 30 sample plots used in this study (blue).

\subsection{Data collection}

\subsubsection{Non-destructive sampling in sample plots}

127 A total of 30 fixed sample plots were selected in 2012 from the Yongfeng forest farm. The plots were located in the eastern section of the study area (Fig. 1). The 30 sampling plots included ten Eucalyptus plantation age groups. In each plot $(0.04 \mathrm{ha}, 20 \mathrm{~m} \times 20 \mathrm{~m})$, we measured the diameter at breast height (DBH) of all living stems $\geq 8 \mathrm{~cm}$ and the tree height $(\mathrm{H})$. In addition, we measured mean plot-level variables, including stand age, density, longitude, latitude, and altitude. 
https://doi.org/10.5194/bg-2020-36

Preprint. Discussion started: 25 February 2020

(C) Author(s) 2020. CC BY 4.0 License.

133 Trees were harvested from standard woods in the 30 fixed sample plots. Three trees with a DBH close to mean DBH of trees in each plot were cut down, for a total of 90 trees harvested from the 30 plots. We then measured the $\mathrm{H}$ and DBH of each harvested tree, as well as the biomass of each organ (foliage, stems, and branches) to obtain the AGB of each harvested tree. Table B.2 in section S2 of the Supplementary Material presents the data for the 90 harvest trees. Details on selection of the standard wood and the cutting process are provided in section S1 of the Supplementary Material.

\subsection{Construction of tree-level allometric models} All analyses were based on the underlying assumption that the relationship between the response and predictor variables in the sample data used to construct the models was the same as the relationship in the entire population. We divided the 90 harvested trees into three age groups (1-2 yr, 3-5 yr, 6-10 yr) for the tree-level allometric models. The allometric models were then applied to each tree in each sample plot according to their age, $\mathrm{DBH}$, and $\mathrm{H}$, thereby producing a true measure of AGB for each sample plot.

\subsection{Construction of plot-level models}

Processing based on model screening was applied to alleviate uncertainty caused by model-dependence and consisted of the four steps shown in Fig. 2. 


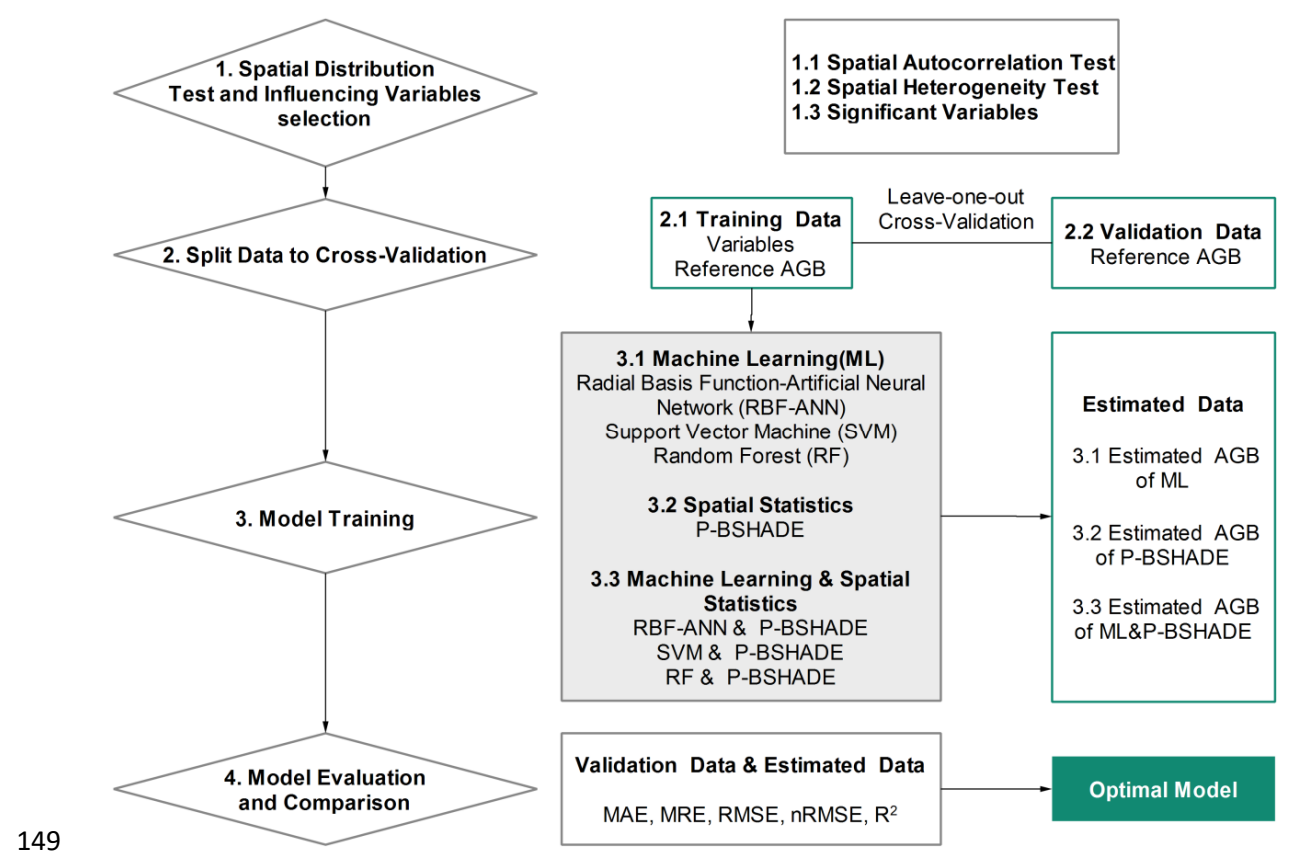

\subsubsection{Selection of variables and analysis of resulting spatial distribution}

To create the plot-level model, we first identified predictor variables. Based on our previous work (Ren

et al., 2017), we selected plot-level environmental covariates including longitude and altitude, and forest attribute variables including forest distribution density, $\mathrm{DBH}, \mathrm{H}$, tree stem volume, and forest age. Pearson's correlation coefficient was used to investigate the correlation between these variables and the true AGB of sample plots. sample plots. We used Moran's I (Cliff and Ord, 1981), a commonly used global spatial autocorrelation index, to evaluate spatial autocorrelation between the true AGBs of sample plots. The spatial stratified heterogeneity (which refers to the within-strata variance being less than the between-strata variance; it is ubiquitous in ecological phenomena, such as AGB) of the true AGB of sample plots was evaluated by using a $q$-statistic generated by applying the GeogDetector model, which is a software tool proposed by Wang et al. (2016) that analyzes spatial variation of the geographical strata of variables. First, we used the K-means algorithm to obtain the strata of true AGB for preprocessing by GeogDetector. Next, 
https://doi.org/10.5194/bg-2020-36

Preprint. Discussion started: 25 February 2020

(C) Author(s) 2020. CC BY 4.0 License.

\subsubsection{Split datasets}

We used the leave-one-out cross-validation method to split the 30 sample plots into 30 sets, with each set containing two groups of data: (1) validation data (the AGB of one plot) and (2) training data (the AGBs and predictor variables of the other 29 plots), see Table B.3. The leave-one-out cross-validation method assumes that, in a dataset containing $n$ samples, each sample serves as a test sample with the other $n-1$ samples serving as training samples. Thus, with $n$ iterations, we can obtain $n$ training datasets and $n$ validation datasets.

\subsubsection{Model training}

Seven models including three machine learning models [Figs. 3(a-3(c)], one spatial statistical model [Fig. 3(d)], and three combined machine learning and spatial statistical models [Figs. 3(a) and 3(d), 3(b) and 3(d), and 3(c) and 3(d)] were developed and trained to predict the AGB of sample plots. The three machine learning models were (a) SVM, (b) RBF-ANN, and (c) RF.

The spatial statistical model (P-BSHADE) required AGB-related variables (reference series). In this case study, we used the reference-plot AGB data as the variables. The allometric model (Qiu et al., 2018) was applied to obtain the AGB of each tree in each sample plot. Next, the reference-plot AGB data consisted of the sum of the AGB of each tree. This method produces the P-BSHADE model shown in Fig. 3(d). For the combined machine learning and spatial statistical models, the reference plot AGB data in P-BSHADE were obtained from the results of the SVM [Fig. 3(a)], the RBF-ANN [Fig. 3(b)], or the RF [Fig. 3(c)]. The three combined models are denoted SVM \& P-BSHADE [Figs. 3(a) and 3(d)], RBF-ANN \& P-BSHADE [Figs. 3(b) and 3(d)], and RF \& P-BSHADE [Figs. 3(c) and 3(d)]. Each model was trained on 30 datasets, yielding a total of 30 predicted AGB datasets for 30 sample plots (see Table B.3, section S2 in the Supplementary Material). 

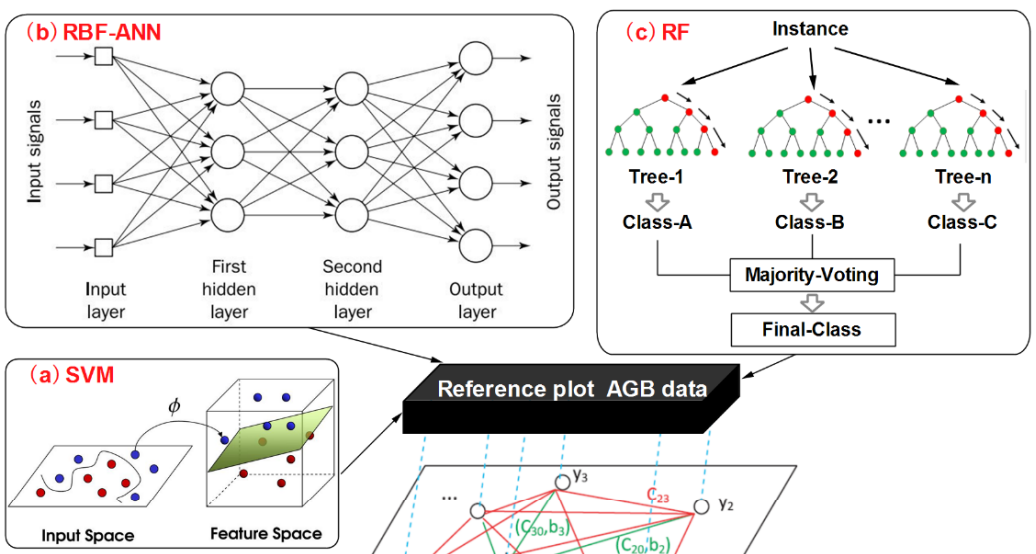

Reference plot AGB data

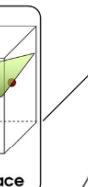

Input Space
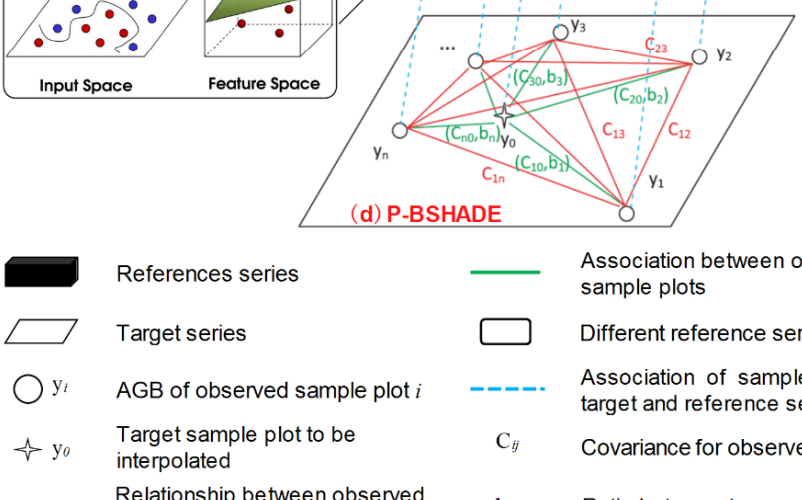

References series

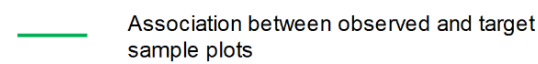

Target series

AGB of observed sample plot $i$

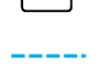

Different reference series

Association of sample plots' AGB between Target sample plot to be interpolated

$C_{i j} \quad$ Covariance for observed sample plot s' AGB

Relationship between observed sample plots

$\mathrm{b}_{i} \quad$ Ratio between two sample plots' AGB

Figure 3. Framework for estimating (a)-(c) the machine learning models, (d) the P-BSHADE model, and the three models that combine machine learning with the P-BSHADE model $(a+d, b+d, c+d)$.

SVM is a method of supervised learning in machine learning and is often used to solve classification problems. The basic principle of SVM is to find a hyperplane in the feature space and separate the positive and negative samples with the minimum misclassification rate (Hearst et al., 1998). RBF-ANN is a three-layer neural network model, which includes an input layer, a hidden layer, and an output layer. The transformation from input space to hidden space is nonlinear, whereas the transformation from hidden space to output space is linear. The function of the hidden layer is to map the vector from the indivisible low-dimensional linear state to the separable high-dimensional linear state, so as to greatly 

Shin, 1994; Xia and Xiu, 2007). RF is a combination of tree predictors such that each tree depends on the values of a random vector sampled independently and with the same distribution for all trees in the forest. RF is an effective tool in prediction. Because of the Law of Large Numbers, RF does not overfit. Injecting the right type of randomness means that RF makes accurate classifiers and regressors (Breiman, 2001).

where $\mathrm{y}_{j}$ is the AGB of the $j$ th sample plot predicted by a machine learning model, $f(\ldots)$ is a machine learning model represented by a function of $x_{j, k}(k=1, \ldots, 4)$; and $x_{j, 1}, x_{j, 2}, x_{j, 3}$, and $x_{j, 4}$ are the central longitude, the mean $\mathrm{DBH}$, the mean $\mathrm{H}$, and the forest age of the $j$ th sample plot, respectively. A specific description of the three machine learning models is given in section S1 of the Supplementary Material.

(2) Spatial statistical model: P-BSHADE

P-BSHADE is an optimal linear unbiased estimation interpolation method based on the assumption of the simultaneous existence of the spatial autocorrelation and heterogeneity of the target object. We use it here to solve the problem of an unrepresentative sample imposed by the spatial location of a convenient sample at the plot level.

The core of the model is to minimize the variances between predicted error and unbiased estimation. The prediction process of the P-BSHADE model requires strong spatio-temporal coordination between the predictive variable (forest AGB of target plots) and the reference series (reference forest AGB of target plots), so as to realize the spatial interpolation of the predictive variable. The model is also a data fusion approach that combines the observed samples with the reference series (related variable).

P-BSHADE is markedly different from the Kriging and Inverse Distance Weighting (IDW) algorithms. Compared with Kriging and IDW, the application of P-BSHADE to forest AGB interpolation has obvious advantages. The spatial distribution of forest AGB is also characterized by spatial autocorrelation and heterogeneity, which have been taken into account in the P-BSHADE model. 
Taking into account spatial heterogeneity can effectively solve the difference in forest AGB distribution caused by different terrain or geographical location. However, Kriging and IDW only consider the spatial correlation between plots. In addition, P-BSHADE considers strongly correlated sample plots as neighboring plots, whereas the Kriging and IDW algorithms consider sites that are close in proximity.

In brief, the P-BSHADE model includes two steps. First, it obtains reference AGB for all sample plots by using the allometric model. Second, it uses the reference AGB of the target sample plot and the true AGB of other sample plots to obtain the weight relationship between the target sample plot and the other sample plots and puts the true AGB of other sample plots and the weights into Eq. (2) to predict the AGB of the sample plots. Therefore, positions and distances between plots do not apply here. The specific mathematical formula for the P-BSHADE model is now described (Hu et al., 2013; Xu et al., 2013).

\section{a. Objective}

The objective is to interpolate the AGB data of the target sample plot by using data acquired from other sample plots. A theoretical description is

$\hat{y}_{j}=\sum_{i=1}^{n} w_{i j} y_{i}$ where $\hat{y}_{j}$ is the AGB of the $j$ th sample plot estimated by the P-BSHADE model $(j=1-30, n=$ 30); $y_{i}$ is the true AGB of the $i$ th sample plot $(i=1-30, n=30) ; w_{i j}$ is the weight (contribution) of the true AGB of the $i$ th sample plot to the AGB to be interpolated of the $j$ th sample plot (when $j=$ $1, i=2,3, \ldots, 30$; when $j=1, i=1,3,5, \ldots, 30) ; w_{i j}$ is calculated by the true AGB of the $i$-th sample plot and the allometric model estimation of the AGB in the $j$-th sample plot.

As expected, the estimates of the two properties in Eq. (2) are unbiased:

$$
E\left(y_{j}\right)=E\left(\hat{y}_{j}\right)
$$

Minimum estimation variance is expressed as

$$
\min _{w}\left[\sigma_{\hat{y}_{j}}^{2}=E\left(\hat{y}_{j}-y_{i}\right)^{2}\right]
$$

where $E$ is the statistical expectation. 


\section{b. Ratio of data from target sample plot to those from other sample plots}

The ratio between data from the target sample plot to those from other sample plots is one of the most important inputs for estimating the $\mathrm{ABG}$ of the target sample plot and is an index of heterogeneity in the AGB spatial distribution. The relationship between data from the target sample plot and from the other sample plots is expressed as

$$
b_{i j} E y_{j}=E y_{i}
$$

In most cases, the AGB of any two plots are not equal, and the relationship between them can be further expressed as the relative bias $b_{i j}$ between the mathematical expectation of $y_{j}$ and $y_{i}$. Considering Eq. (2), Eq. (5) can be written as

$$
\sum_{i=1}^{n} w_{i j} b_{i j}=1
$$

This equation is generally valid for nonhomogeneous conditions. Clearly, the determination of $b_{i j}$ requires calculating the coefficients $w_{i j}(i=1, \ldots, n, j=1, \ldots, n)$, which is addressed in the following section.

\section{c. Weight estimation}

The main challenge in estimation is finding the weights $w_{i j}$ that satisfy the unbiased condition and that minimize estimation variance:

$$
\sigma_{\hat{y}_{j}}^{2}=E\left(\hat{y}_{j}-y_{i}\right)^{2}=C\left(\hat{y}_{j} \hat{y}_{j}\right)+C\left(y_{i} y_{i}\right)-2 C\left(\hat{y}_{j} y_{i}\right)
$$

These weights can be calculated by minimizing the estimation variance and taking unbiasedness into account:

$$
\left[\begin{array}{cccc}
C\left(y_{1} y_{1}\right) & \cdots & C\left(y_{1} y_{n}\right) & b_{1 j} \\
\vdots & \ddots & \vdots & \vdots \\
C\left(y_{n} y_{1}\right) & \cdots & C\left(y_{n} y_{n}\right) & b_{n j} \\
b_{1 j} & \cdots & b_{n j} & 0
\end{array}\right]\left[\begin{array}{c}
w_{1 j} \\
\vdots \\
w_{n j} \\
\mu
\end{array}\right]=\left[\begin{array}{c}
C\left(y_{1} y_{j}\right) \\
\vdots \\
C\left(y_{n} y_{j}\right) \\
1
\end{array}\right]
$$

where $\mu$ is a Lagrange multiplier. The minimized variance in the estimation error can then be written as

$$
\sigma_{y}^{2}=\sigma_{y_{i}}^{2}+\sum_{i=1}^{n} \Sigma_{k=1}^{n} C\left(y_{i} y_{k}\right)-2 \sum_{i=1}^{n} w_{i j} C\left(y_{i} y_{j}\right)+2 \mu\left(\sum_{i=1}^{n} w_{i j} b_{i j}-1\right)
$$


https://doi.org/10.5194/bg-2020-36

Preprint. Discussion started: 25 February 2020

(c) Author(s) 2020. CC BY 4.0 License.

The P-BSHADE model is a geospatial model because it has the following characteristics:

1. The P-BSHADE model is mainly based on the assumptions of spatial autocorrelation and spatial

heterogeneity of forest AGB. Therefore, before using P-BSHADE, we first applied the statistical test of these two theoretical hypotheses (spatial autocorrelation test and spatial differentiation test) for forest AGB.

2. The prediction process of the P-BSHADE model requires strong spatio-temporal coordination between the predictive variable (forest $\mathrm{AGB}$ of target plots) and the reference sequence (reference forest AGB of target plots), so as to spatially interpolate the predictive variable.

3. P-BSHADE is an optimal linear unbiased estimation interpolation method that considers temporal and spatial heterogeneity. Spatial autocorrelation and heterogeneity of AGB data can be added into the model based on prior knowledge (reference AGB data), following which the linear unbiased optimal estimation of the target-plot AGB can be obtained by correcting data from a convenient sample plot. used [see 2.4.3(2)b section]. In the P-BSHADE model, this ratio plays a very important role in estimating the forest AGB of the target plots. This ratio is a manifestation of the spatial heterogeneity of AGB data. P-BSHADE takes into account the reality of the spatial distribution of AGB data and emphasizes that the spatial distribution of AGB data is heterogeneous.

(3) Combination of machine learning and spatial statistical models investigates whether their combination can improve the accuracy of forest AGB estimates. Therefore, P-BSHADE was separately integrated with the three machine learning methods (SVM, RBF-ANN, and RF) to form three combined models (SVM \& P-BSHADE, RBF-ANN \& P-BSHADE, and RF \& P-BSHADE). The reference AGBs of the 30 sample plots were replaced by the estimates produced by 
where $\hat{y}_{j}$ is the estimated AGB of the $j$ th sample plot using the combined model $(j=$ $1,2, \ldots, 30, n=30) ; y_{i}$ is the true AGB of the $i$ th sample plot $(i=1,2, \ldots, 30, n=30) ; w_{i j}$ is the contribution in weight of the $i$ th true AGB of the sample plot to the $j$ th sample plot AGB to be interpolated (when $j=1, i=2,3, \ldots, 30$; when $j=1, i=1,3,5, \ldots, 30$ ); $w_{i j}$ is calculated by using the true AGB of the $i$ th sample plot and the machine learning estimate of the AGB of the $j$ th sample plot. A detailed description of the combined models and the algorithm formulas is presented in section S1 of the Supplementary Material.

\subsubsection{Model evaluation and comparison}

To evaluate the accuracy of the AGB estimates of the seven models (SVM, RBF-ANN, RF, P-BSHADE, SVM \& P-BSHADE, RBF-ANN \& P-BSHADE, and RF \& P-BSHADE), the AGB results were compared to the reference AGBs of the sample-plot groups (AGB group M in Table B.3). We calculated four performance indicators, as given by Eqs. (11)-(14) [mean absolute error (MAE), mean relative error (MRE), root mean square error (RMSE), and normalized root mean square error (nRMSE)]:

$\operatorname{MAE}=\left(\sum_{i=1}^{n}\left|y_{i}^{p}-y_{i}\right|\right) / n$

$\operatorname{MRE}=\left(\sum_{i=1}^{n}\left|y_{i}^{P}-y_{i}\right|\right) /\left(y_{i} \times n\right)$

$\operatorname{RMSE}=\sqrt{\left(\sum_{i=1}^{n}\left(y_{i}^{p}-y_{i}\right)^{2}\right) / n}$

nRMSE $=\frac{\sqrt{\left(\sum_{i=1}^{n}\left(y_{i}^{p}-y_{i}\right)^{2}\right) / n}}{\overline{y_{l}}}$

where $y_{i}^{p}$ is the predictive value of the different models, $y_{i}$ is the AGB of the $i$ th sample plot, and $n$ is the number of training datasets.

We then used the calculated MAE, MRE, RMSE, and nRMSE to identify the optimal model.

\subsubsection{Robustness of combined models}

To evaluate the robustness of the combined machine learning and spatial statistical models, we selected 22 independent sample plots (see details in S1 and S3 of the Supplementary Material) and made nondestructive measurements of each tree in July 2019. We repeated the workflow used for constructing the plot-level model and evaluated the models. We then evaluated whether the combined 
https://doi.org/10.5194/bg-2020-36

Preprint. Discussion started: 25 February 2020

(C) Author(s) 2020. CC BY 4.0 License.

(MAE, MRE, RMSE, and nRMSE).

\subsection{Model application and upscaling}

We treated the irregular polygon forest patches (2980 patches) of the Forest Management and Planning Inventory (FMPI) as a homogenous sample plot and used the optimal plot-level model to upscale forest AGB (see section S1 of the Supplementary Material). We then compared the upscaled forest AGB with the AGB map obtained from the allometric model and calculated the MRE of AGB between the two methods (see Eq. A.15 in section S1 of the Supplementary Material).

\section{Results}

\subsection{True AGB of sample plots}

The true AGB for the 30 sample plots ranged from 1.02 to $135.79 \mathrm{Mg} \cdot \mathrm{ha}^{-1}$, with an average value of 47.34 Mg $\cdot \mathrm{ha}^{-1}$ and a standard deviation of $34.46 \mathrm{Mg} \cdot \mathrm{ha}^{-1}$. The coefficients of variation of the AGB for all the sample plots and for the 10 age categories were 0.73 and $0.07-0.37$, respectively.

\subsection{Spatial distribution test and the selection of variables}

\subsubsection{The effect of different variables}

Figure 4 shows the correlation-coefficient matrix of variables. The following variables were strongly correlated with AGB: longitude $(r=-0.56)$, DBH $(r=0.79), H(r=0.84)$, trunk volume $(r=0.86)$, and forest age $(r=0.82)$. Timber volume and stem volume were both estimated based on $\mathrm{H}$ and $\mathrm{DBH}$, so they were excluded as covariates for the AGB plot-level models. To summarize, four variables (longitude, $\mathrm{DBH}, \mathrm{H}$, and forest age) were selected as covariates for the AGB plot-level models of the Eucalyptus forest in the Nanjing region. Table B.4 in section S2 of the Supplementary Material lists the statistical descriptions of these covariates and the AGB statistics for the 30 sample plots. 
https://doi.org/10.5194/bg-2020-36

Preprint. Discussion started: 25 February 2020

(c) Author(s) 2020. CC BY 4.0 License.

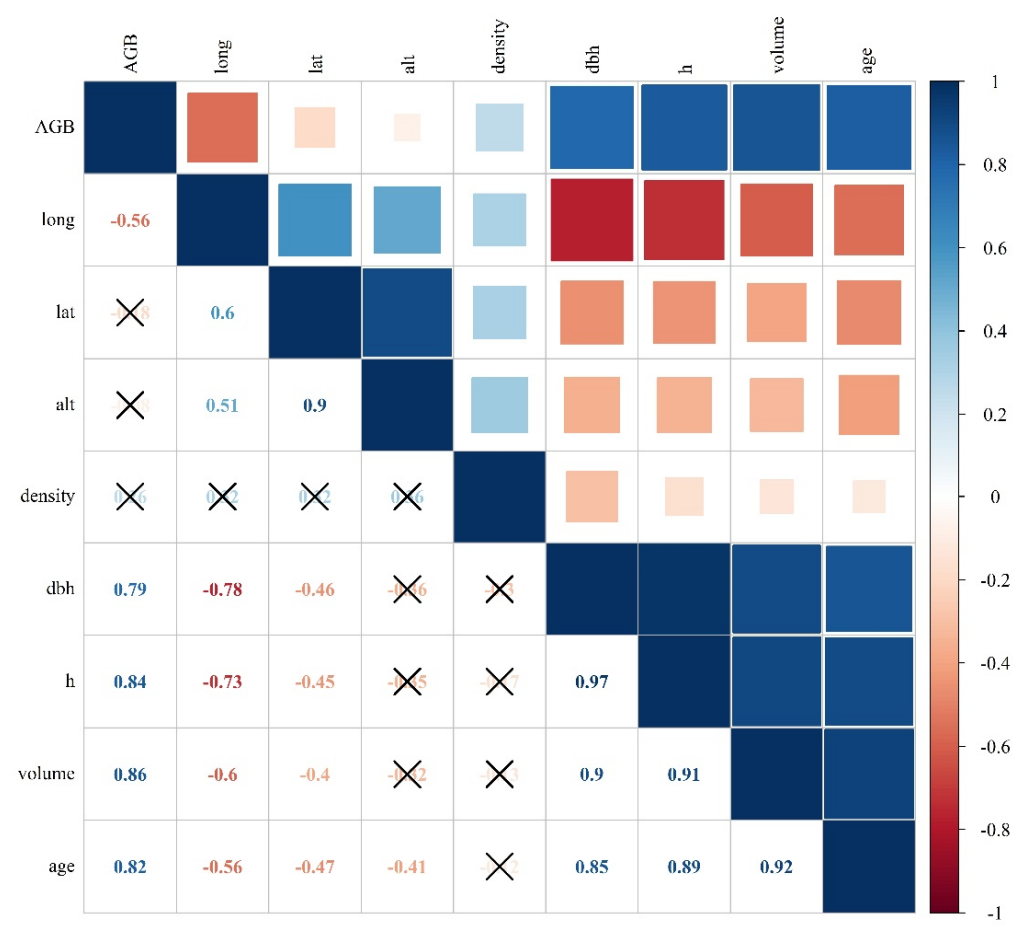

Figure 4. Pearson's correlation coefficients between AGB and other variables represented by numbers and squares. Negative (red) numbers indicate that the corresponding variables are negatively correlated and are colored in red, whereas positive (blue) numbers represent positive correlations. Larger absolute numbers are indicated by darker colors, larger squares indicate stronger correlations, and the symbol " $\times$

\subsubsection{Spatial autocorrelation test}

The spatial distribution of the true AGBs of the 30 sample plots displayed a pattern of aggregation (see red regions in Fig. C.1, section S3 of the Supplementary Material and Table 1). In addition, because less than $1 \%$ of the AGB data were randomly distributed (see blue regions in Figs. C.1 and S3 of the Supplementary Material and Table 1), the possibility of an aggregated distribution was greater than that of random distribution. Furthermore, the null hypothesis was significantly rejected $(p<0.01)$. These results suggest that the spatial distribution of the AGB data displays aggregation and a pattern of strong 
https://doi.org/10.5194/bg-2020-36

Preprint. Discussion started: 25 February 2020

(c) Author(s) 2020. CC BY 4.0 License.

Table 1. Spatial autocorrelation and heterogeneity test.

\begin{tabular}{|c|c|c|c|c|}
\hline \multicolumn{2}{|c|}{ Spatial autocorrelation } & \multicolumn{3}{|l|}{ Spatial heterogeneity } \\
\hline Items & Values & Factors & $q$ value & $p$ value \\
\hline & & AGB & 0.87 & $<0.01$ \\
\hline \multirow[t]{2}{*}{ Moran I } & 0.36 & & & \\
\hline & & Longitude, long & 0.38 & $<0.01$ \\
\hline \multirow[t]{2}{*}{$z$-score } & 4.78 & Diameter at breast height, DBH & 0.54 & $<0.01$ \\
\hline & & Tree height, $\mathrm{H}$ & 0.63 & $<0.01$ \\
\hline \multirow[t]{2}{*}{$p$-value } & 0.00 & & & \\
\hline & & Age & 0.92 & $<0.01$ \\
\hline
\end{tabular}

\subsubsection{Spatial heterogeneity test}

As shown in Table 1, the true AGBs of the sample plots were divided into three strata by using $k$-means clustering. We then ran the GeogDetector model and obtained a $q$ value of 0.87 and a $p$ value less than 0.01 . These results indicate that the within-layer variances were far less than the sum of variances among different strata. The results also suggest that the reference AGBs of the 30 sample plots were associated with obvious spatially stratified heterogeneity.

\subsection{Performance of plot-level models}

We developed seven models for estimating AGB: three machine learning models (SVM, RBF-ANN, and RF), one spatial statistical model (P-BSHADE), and three combined models that integrated each machine learning method with the spatial statistical method (SVM \& P-BSHADE, RBF-ANN \& P-BSHADE, and RF \& P-BSHADE). Furthermore, we used the leave-one-out cross-validation method to split the datasets and evaluated the prediction performance of these seven methods based on the indicators MAE [Fig. 5(a)], MRE [Fig. 5(b)], RMSE [Fig. 5(c)], and nRMSE [Fig. 5(d)]. 
(a)

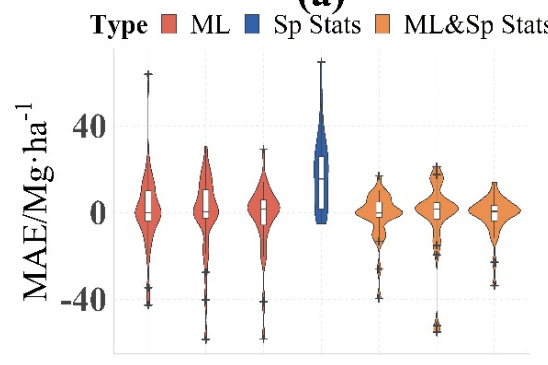

(c)

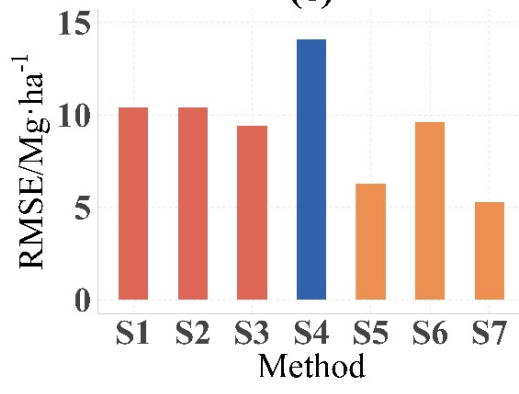

(b)

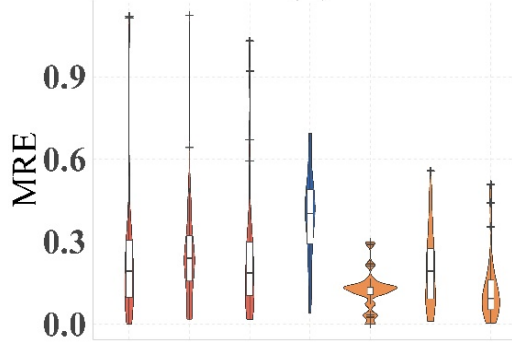

(d)

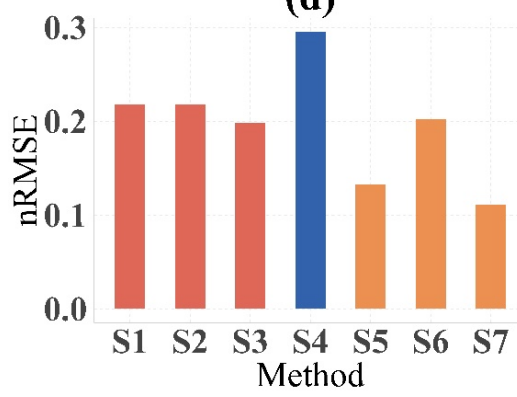

Figure 5. Prediction performance of the seven different models. (a) MAE and (b) MRE are presented as boxplots for each prediction method, with the median (black horizontal line in the box), inter-quartile range ( $25 \%-75 \%$ in the box), the range $5 \%-95 \%$ (whiskers), and outliers (asterisks) labeled (S1=SVM, S2=RBF-ANN, S3=RF, S4=P-BSHDE, S5=SVM \& P-BSHDE, S6=RBF-ANN \& P-BSHDE, S7=RF \& P-BSHDE, ML=machine learning, Sp Stats=Spatial statistics). Histogram distributions of RMSE and nRMSE for each prediction method are presented in panels (c) and (d), respectively.

The forest AGB estimates obtained by the three machine learning methods were significantly more accurate than those obtained by the spatial statistical method. The performance indicators for P-BSHADE were $\mathrm{MAE}=18.37 \mathrm{Mg} \cdot \mathrm{ha}^{-1}, \mathrm{MRE}=39.13 \%, \mathrm{RMSE}=14.08 \mathrm{Mg} \cdot \mathrm{ha}^{-1}$, and $\mathrm{nRMSE}=29.57 \%$, whereas those for the machine learning methods covered the following ranges: MAE 10.16-12.15 $\mathrm{Mg} \cdot \mathrm{ha}^{-1}$, MRE 24.79\%-26.69\%, RMSE 9.43-10.39 Mg $\mathrm{ha}^{-1}$, and nRMSE 19.80\%-21.82\%.

Among the three machine learning methods, the accuracy of RF was highest. The four evaluation indexes $\left(\mathrm{MAE}=10.16 \mathrm{Mg} \cdot \mathrm{ha}^{-1}, \mathrm{MRE}=25.93 \%, \mathrm{RMSE}=9.43 \mathrm{Mg} \cdot \mathrm{ha}^{-1}\right.$, and $\left.\mathrm{nRMSE}=19.80 \%\right)$ were 
https://doi.org/10.5194/bg-2020-36

Preprint. Discussion started: 25 February 2020

(c) Author(s) 2020. CC BY 4.0 License. $\left(\mathrm{MAE}=11.17-12.15 \mathrm{Mg} \cdot \mathrm{ha}^{-1}, \mathrm{MRE}=24.79 \%-26.69 \%, \mathrm{RMSE}=10.39-10.39 \mathrm{Mg} \cdot \mathrm{ha}^{-1}\right.$, and $\mathrm{nRMSE}=$ $21.82 \%)$. Finally, the combination of machine learning and spatial statistical models produced smaller MAE (5.68-10.14 Mg.ha $\left.{ }^{-1}\right)$, MRE (12.47\%-20.49\%), RMSE (5.30-9.63 Mg.ha $\left.{ }^{-1}\right)$, and nRMSE

404

405 $(11.13 \%-20.23 \%)$ than the single machine learning methods. Of the three combined methods, RF \& P-BSHADE produced the highest accuracy with the smallest MAE $\left(5.68 \mathrm{Mg} \cdot \mathrm{ha}^{-1}\right)$, a modest MRE (12.97\%), and the smallest RMSE (5.30 Mg $\left.\cdot \mathrm{ha}^{-1}\right)$ and nRMSE (11.13\%). In contrast, RBF-ANN \& P-BSHADE had the highest MAE (10.14 Mg·ha ${ }^{-1}$ ), MRE (20.49\%), RMSE (9.63 Mg ha ${ }^{-1}$ ), and nRMSE (20.23\%). Compared with the RF model, the RF\&P-BSHADE model led to a reduction of the cross-validated prediction error of $43.80 \% \sim 50.00 \%$ ( $44.08 \%$ for MAE, $50.00 \%$ for MRE, and $43.80 \%$ for RMSE and nRMSE).

We also explored the relationship between the observed and predicted AGBs in terms of cross-validation results (Fig. 6). The quantity $\mathrm{R}^{2}$ was calculated for the linear regression model applied to the observed and predicted AGBs; $\mathrm{R}^{2}$ for every model was greater than 0.9. Although P-BSHADE had the highest $\mathrm{R}^{2}$, its distribution of dots in Fig. 6(d) differed quite significantly from the 1:1 line. Of the seven models, the accuracy of RF \& P-BSHADE was the highest and the distribution of dots in Fig. 6(g) was closest to the 1:1 line. Therefore, we concluded that RF \& P-BSHADE was the optimal model. 
(a)

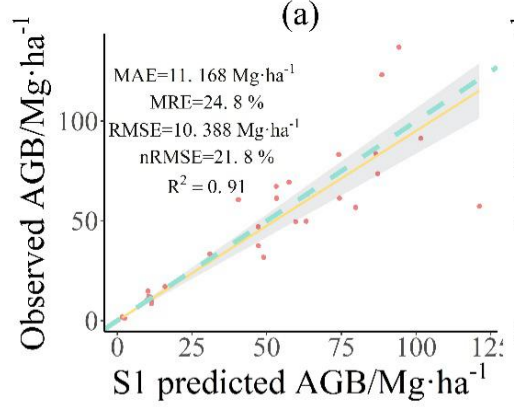

(c)
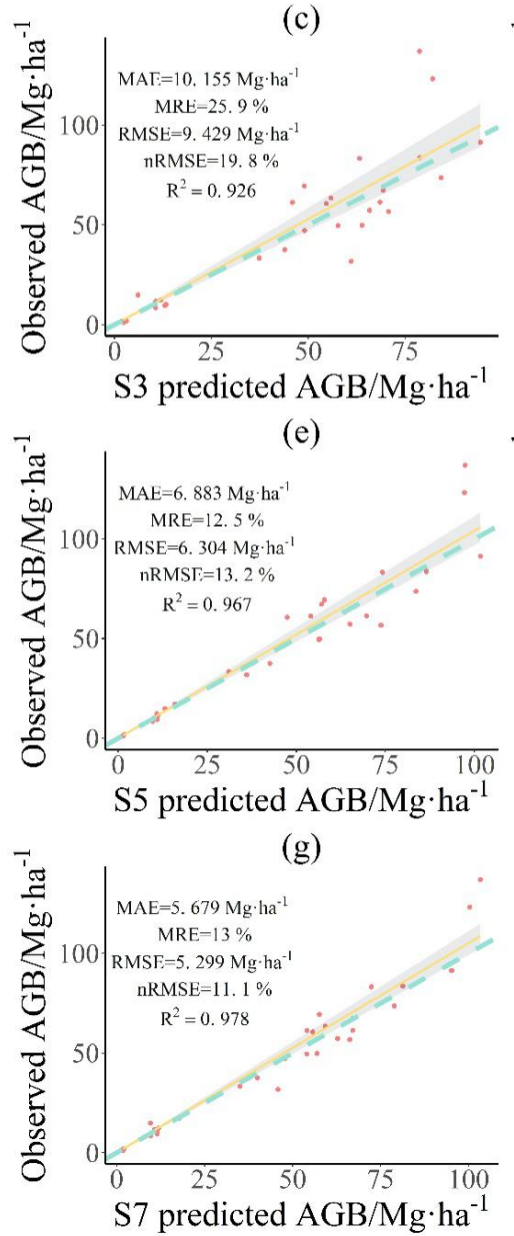

(b)

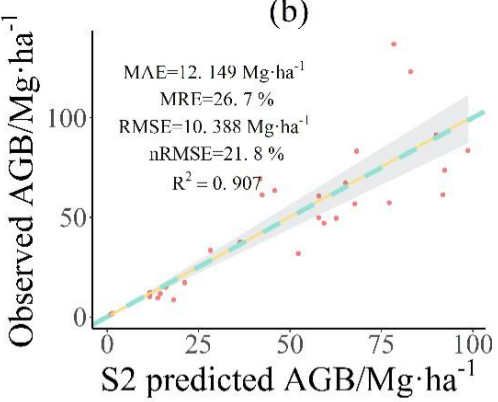

(d)

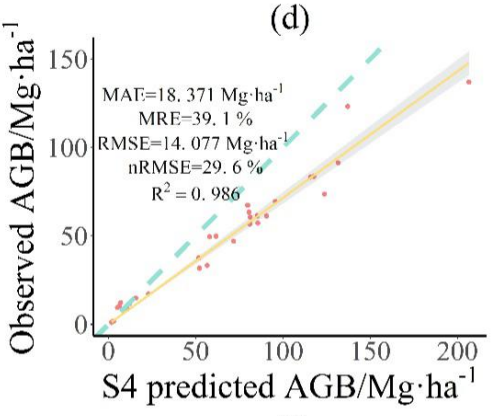

(f)

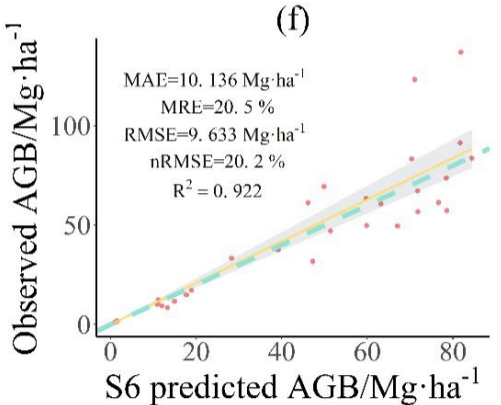


423 We compared three machine learning methods with three corresponding combined machine learning periods, 2012 and 2019 (Fig. 7). The results suggest that the combined models improved the accuracy of single machine learning models during both years. This suggests that the combined methods are robust.

(a)

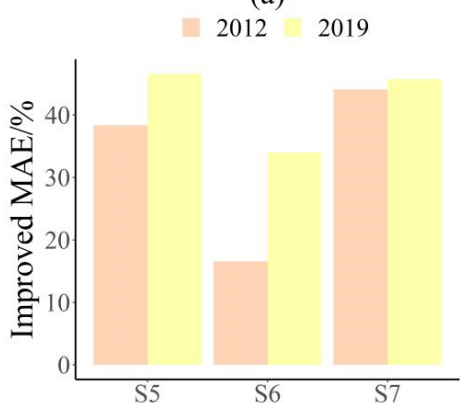

(c)

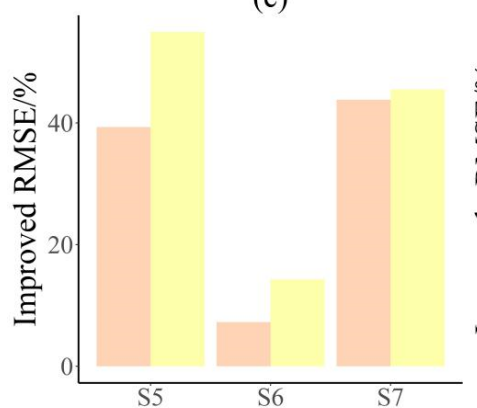

(b)

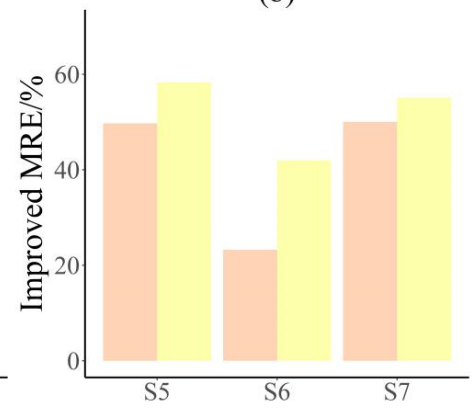

(d)

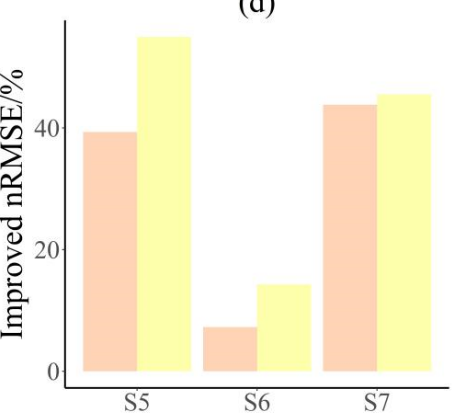

428

Figure 7. The improvement in accuracy assessment indexes of three combined machine learning and spatial statistical methods by comparison with three corresponding machine learning methods. Panels (a)-(d) show the MAE, MRE, RMSE, and nRMSE, respectively; S1-S5 represents RMSE comparison of S5 with S1, S2-S6 represents RMSE comparison of S6 with S2, and S3-S7 represents RMSE comparison of S7 with S3 (S1=SVM, S2=RBF-ANN , S3=RF, S4=P-BSHDE , S5=SVM \& P-BSHDE, S6=RBF-ANN \& P-BSHDE, S7=RF \& P-BSHDE).

Figure C.3 in section S3 of the Supplementary Material shows the spatial distribution of AGBs 
predicted by the RF \& P-BSHADE model. The predicted AGBs were 7.54-89.93 $\mathrm{Mg} \cdot \mathrm{ha}^{-1}$, with an average of $41.21 \mathrm{Mg} \cdot \mathrm{ha}^{-1}$, a median of $43.53 \mathrm{Mg} \cdot \mathrm{ha}^{-1}$, a standard deviation of $18.83 \mathrm{Mg} \cdot \mathrm{ha}^{-1}$, and a coefficient of variation of $45.69 \%$. The total AGB of the Nanjing region (2980 forest patches) estimated by RF \& P-BSHADE was $122812.1 \mathrm{Mg}$, whereas that estimated by the allometric model was $123021.5 \mathrm{Mg}$. The percent difference in total AGB between the two methods was $0.17 \%$. Meanwhile, the AGB MRE between the two methods ranged from $0.04 \%$ to $99.8 \%$, with an average of $19.93 \%$.

\section{Discussion}

we developed, evaluated, and compared the accuracy and performance of three different machine learning models [support vector machine (SVM), random forest (RF), and the radial basis function artificial neural network (RBF-ANN)] in this study, which contains one spatial statistics model (P-BSHADE) and three combinations thereof (SVM \& P-BSHADE, RF \& P-BSHADE, ANN \& P-BSHADE) on forest AGB estimates. Those findings suggested that the combined models, especially the RF \& P-BSHADE model, could improve the accuracy of plot-level AGB estimates and could reduce the uncertainty of plot-level AGB estimates, owing to its integrated the theoretical advantages of machine learning and spatial statistics.

\subsection{Significance of the optimal AGB model at the plot-level}

In the past, ecologists converted AGB estimates from forest sample plots into regional AGB estimates by scaling up from the tree-level to the regional scale (Malhi et al., 2004). Plot-level AGB models therefore link tree-level AGB models to regional-scale AGB models. Research by Chen et al. (2015) found that ignoring the uncertainty of plot-level models increased the total uncertainty of pixel-level estimates by $6 \%$. In addition, Marvin et al. (2014) found that the distribution pattern of most AGB is either non-Gaussian, skewed, or multi-modal, especially in tropical and subtropical regions. Different intensity and direction of factors are coupled together, resulting in high heterogeneity and clear nonlinearity in the spatial distribution of forest AGB.

Here, we integrated the advantages of machine learning and spatial statistics at the plot level (the key scale linking the tree-level scale to the landscape scale) to construct a plot-level AGB model for a 
https://doi.org/10.5194/bg-2020-36

Preprint. Discussion started: 25 February 2020

(c) Author(s) 2020. CC BY 4.0 License.

subtropical region. The approach provides a high-precision plot-level AGB model whose estimates can be compared with those obtained from remote sensing, ground observations, and model simulations. It also provides a foundation for making informed forest management decisions (e.g., the method enables quantitative evaluation of carbon emissions from deforestation). Combining the advantages of machine-learning-based quantification of $\mathrm{AGB}$ and the complex nonlinear relationships between multiple environmental covariates, in conjunction with the P-BSHADE model, allows the spatial autocorrelation and heterogeneity of multiple environmental covariates to be incorporated into the model. In addition, the sample points are subsequently rectified, thus leading to the best linear unbiased estimate of the target plots.

\subsection{Model comparisons}

\subsubsection{Machine learning outperforms the spatial statistical model}

Regarding the AGB plot-level models, the machine learning methods outperformed the spatial statistical method (P-BSHADE) in terms of prediction accuracy. This may be because machine learning offers an array of supervised learning models capable of relating forest AGB to multi-variables, including forest variables and environmental variables, via complex, potentially nonlinear functional relationships. Machine learning models appear adept at tackling high-dimensional problems, particularly in areas where effective algorithms are lacking and where programs must dynamically adapt to changing conditions (Görgens et al., 2015; Latifi et al., 2010; Stojanova et al., 2010). In addition, the P-BSHADE model yielded negative weights between a small number of plots, which might introduce a slight degree of uncertainty into the results (Xu et al., 2013). Our results were consistent with those of Povak et al. (2014) and Li et al. (2011), who found that a machine learning method (RF) outperformed the spatial statistical method (e.g., Geographically Weighted Regression, Inverse Distance Weighting ) in terms of prediction accuracy.

\subsubsection{Why a combined model outperforms a single machine learning or spatial statistical model}

As expected, the prediction accuracies of the combined methods were higher than those of any single method (either machine learning or spatial statistical). This may due to the advantages of machine learning, which can compensate for the inherent defects of the P-BSHADE model, and vice versa. 
autocorrelation and spatial heterogeneity of the distribution of the target objects, not only to solve the difference between target objects caused by the different terrain or geographical location but also to solve the problem of strong correlation between target objects with remote geographical locations due to similar terrain condition. (2) The P-BSHADE model calculates the covariance between objects by using a reference sequence between objects (which means the reference AGB data between plots in our study). This method is more reliable because it avoids the second-order stationary hypothesis (i.e., when using the Kriging algorithm, semi-variograms need this hypothesis), which does not correspond with the actual situation. (3) P-BSHADE regards strongly correlated plots as neighboring plots. However, the P-BSHADE model is also handicapped by the fact that the founding assumption does not conform to reality. The assumption is that estimated AGB is accurate in all sampling plots except the target sampling plot. In other words, the premise behind using only the P-BSHADE model is that the reference AGB data is accurate or strongly correlated with AGB. In reality, the AGB of each sampling plot has a varying degree of uncertainty because it is obtained from the allometric model. Since the P-BSHADE model combined with machine learning uses the results optimized by machine learning as the reference series, it further improves the accuracy of AGB mapping.

Machine learning also has its advantages and disadvantages. As we described in the previous section (4.2.2), machine learning has the advantage of being able to handle complex, potentially nonlinear relationships between forest $\mathrm{AGB}$ and other variables. However, the initial samples of machine learning are randomly selected, which may lead to differences in the results of each operation of the model. In addition, machine learning uses the average value of all regression trees in the calculation, which may result in overestimating the lower value and underestimating the higher value. As opposed to machine learning, the P-BSHADE model takes into account the spatial autocorrelation and spatial heterogeneity of forest AGB and of environmental covariates, and the bias of the observed values of the sampling plots, which corresponds more to actual situations. A combined model takes the result of machine learning as the reference series of P-BSHADE, so that the fitting process of the combined model takes spatial relationships more into account than is the case for the single machine learning model. The end result is improved accuracy.

Machine learning models or the P-BSHADE model have been used to model the uncertainty of temperature measurements obtained by weather stations (Fassnacht et al., 2014; Paul et al., 2016; Xu et 
al., 2013). However, the methods used in these studies were adopted independently. Conversely, the combination of machine learning and spatial statistics can improve the prediction accuracy of AGB maps, which in turn can be used as criteria for improving the accuracy of LiDAR remote-sensing technology and the results of ecological process models. Eventually, these improvements can promote process-oriented projects that require dynamic AGB predictions for large-scale forests in different forest management scenarios.

In addition, we compared the prediction accuracy of AGB mapping obtained by the combined spatial statistical and machine learning models with that reported by recent studies using AGB plot-level models. In the current literature on remote-sensing estimation of forest AGB, nRMSE, RMSE, and R ${ }^{2}$ were commonly used as indexes for evaluating the prediction performance of models affected by research sample size, data type, and forecasting methods (Fassnacht et al., 2014). In contrast, the present study used four conventional indexes for evaluating prediction performance: nRMSE, RMSE, MAE, and MRE. The criterion for model selection is to choose indexes summarized from sample prediction (such as nRMSE), rather than choosing the goodness-of-fit $\mathrm{R}^{2}$ (Babcock et al., 2015). Based on calculated nRMSE indexes, the AGB prediction accuracy of the combined RF \& P-BSHADE model $(11.13 \%)$ was higher than that obtained by Babcock et al. (2015) (33.91\%) in Colorado, USA. In that study, the authors used a combination of airborne LiDAR, a forest inventory database, and a Bayesian spatial hierarchical framework model and introduced spatial random effects to compensate for the residual spatial dependence and non-stationary model covariates. The AGB prediction accuracy of the method developed in the current work was also greater than that obtained by Ioki et al. (2014) $(\mathrm{nRMSE}=26 \%$ ) in northern Borneo using a stepwise linear regression model with airborne LiDAR and a ground survey. Furthermore, it exceeded the accuracy obtained by Hansen et al. (2015) in the tropical submontane rain forest (34.4\%) using fusion maps of multi-source databases combined with multiple regression analysis. Our prediction accuracy is close to that obtained by Kim et al. (2016) (9.2\%) who studied an intact tropical rain forest by using a voxel-based method based on airborne LiDAR in conjunction with field monitoring in Brunei. Our combined methods produce very small RMSE for the prediction accuracy of AGB, which we attribute to the following reasons: (1) The true AGBs of the 30 sample plots were calculated from each tree by using an allometric model constructed from the 90 most accurate harvested trees. There were no differences in the range of true values. (2) Machine learning 
methods were used to quantify the complex nonlinear relationship between AGB and multiple environmental covariates. (3) We applied a spatial statistical method based on the hypothesis of spatial heterogeneity. Although the nRMSE index was calculated by different studies using different datasets and prediction methods in different locations, most studies agreed that nRMSE was the most commonly used indicator for measuring the AGB prediction errors of plot-level models and for calculating the true AGB of forest sample plots. In contrast to other studies, our work reflects not only a focus on subtropical forests but also the methodological differences in uncertainty mitigation, especially in terms of comprehensively addressing the sources of uncertainty caused by multiple spatial and environmental covariates.

\subsubsection{Why RF \& P-BSHADE method outperforms other combined methods}

The three combined machine learning and spatial statistical methods produced more accurate AGB predictions than any individual method. The accuracy of the RF \& P-BSHADE and SVM \& P-BSHADE methods were significantly higher than that of the individual methods, but the RBF-ANN \& P-BSHADE method was only slightly higher. The accuracies of the combined methods depend on the accuracy of the reference series (machine learning predicted result) (Xu et al., 2013). In other words, the higher the accuracy of the predicted machine learning results, the higher the accuracy of the combined method. Therefore, the different improvements offered by the three combined methods may be attributed to the following two mechanisms: (1) the RF and SVM models are easier to use and optimize than RBF-ANN (Raczko and Zagajewski, 2017). RBF-ANN is sensitive to hyper-parameters and usually requires optimized parameters to obtain better fitting results. However, in the present study, we used no optimized algorithms, such as genetic algorithms, to obtain parameters in the machine learning model. Furthermore, the number of training samples determines the number of nodes in the hidden layer of the RBF-ANN model, and the number of nodes significantly affects the prediction accuracy. With only 30 training samples used in this study, the combined approach may have been unable to strongly improve prediction accuracy. (2) RBF-ANN is more suitable for nonlinear stochastic dynamic systems (Elanayar and Shin, 1994), whereas the relationship between AGB and environmental covariates in this study is likely a monotonically increasing function. 


\subsection{Comparing upscaling of RF\&P-BSHADE with allometric model}

We used FMPI data to upscale the optimal plot-level AGB model from plot level to region scale. Because the allometric model offers a fast and simple calculation method, it has been used in many studies as the basis for determining the benchmark map. Nevertheless, spatial heterogeneity caused by multiple environmental covariates is not considered in the allometric model because potential errors in the AGB estimate may be propagated and affect the accuracy of the regional AGB map. Although we regarded the FMPI patches as homogeneous study units in the present study, the area of the forest patches is significantly larger than that of the sample plots. Upscaling results will thus have large uncertainties (see Figs. C.4, S3 of Supplementary Material) (Chen et al., 2015). The current study finds that the relative percent difference in total AGB between RF \& P-BSHADE and the allometric model was $0.17 \%$. Meanwhile, the relative error (RE) in AGB between the two models ranged from $0.04 \%$ to $99.8 \%$ with a MRE of $19.93 \%$. This suggests that the two methods are similar in terms of overall estimates of AGB but that the local spatial distribution of AGB differs. Differences in AGB spatial distribution have been reported in many studies of AGB maps. Babcock et al. (2015) asserted that the main reasons for the differences in the spatial distribution of AGB maps between different methods include the following: (1) The structural framework of different research methods and schemes cannot truly reflect actual forest growth. (2) The model is usually a simplification of an ecological process and ignores spatial heterogeneity at the regional scale. (3) The model does not consider the influence of multiple environmental covariates (vegetation, topography, and others) on forest growth in the region.

\section{Conclusions}

This paper proposes a method to integrate the advantages of machine learning and spatial statistics, different datasets, and multiple environmental covariates to improve the accuracy of plot-level AGB-estimation models. In this study, we explored the prediction performance of different AGB models and found that the model that combines the Random Forest and P-BSHADE models substantially improved estimates of forest AGB. Although data from the sample plots and harvested trees were collected only from Eucalyptus forests in the Nanjing region of China, the proposed model and the associated results can provide references for AGB mapping in other countries and in different types of tropical forests. 
https://doi.org/10.5194/bg-2020-36

Preprint. Discussion started: 25 February 2020

(c) Author(s) 2020. CC BY 4.0 License.

Data availability.

All data are included in the paper and Supplement.

\section{Author Contributions}

Y.R. designed the study. X.Z. carried out the data collection. S.D. carried out the analyses and visualized the data. X.Z. and S.D. wrote the manuscript with help from Y.R. L.G., C.X., S.Z., Q.C., and X.W. provided technical advice and guidance throughout the project implementation and paper-writing stages. S.D. and X.Z. contributed equally to this work.

\section{Competing interests.}

The authors declare that they have no conflict of interest.

\section{Acknowledgments}

Shaoqing Dai and Xiaoman Zheng contributed equally to this work and should be considered as co-lead authors. This work was supported by National Science Foundation of China (Grants No. 31670645, No. 31972951, No. 31470578, No. 31200363, No. 41801182, and No. 41807502), National Social Science Fund (17ZDA058), the National Key Research Program of China (2016YFC0502704), Fujian Provincial Department of S\&T Project (Grants No. 2016T3032, No. 2016T3037, No. 2016Y0083, No. 2018T3018, No. 2019J01136, and No. 2015Y0083), the Strategic Priority Research Program of Chinese Academy of Sciences (XDA23020502), the Ningbo Municipal Department of Science and Technology(2009C10056), the Xiamen Municipal Department of Science and Technology (Grants No. $3502 Z 20130037$ and No. 3502Z20142016), the Key Laboratory of Urban Environment and Health of CAS (KLUEH-C-201701), the Key Program of the Chinese Academy of Sciences (KFZDSW-324), and the Youth Innovation Promotion Association CAS (2014267). We are grateful to the anonymous reviewers for their constructive suggestions. 


\section{References}

Andersen, H.-E., Reutebuch, S. E., McGaughey, R. J., d'Oliveira, M. V. N., and Keller, M.: Monitoring selective logging in western Amazonia with repeat lidar flights, Remote Sensing of Environment, 151, 157-165, 10.1016/j.rse.2013.08.049, 2014.

Babcock, C., Finley, A. O., Bradford, J. B., Kolka, R., Birdsey, R., and Ryan, M. G.: LiDAR based prediction of forest biomass using hierarchical models with spatially varying coefficients, Remote Sensing of Environment, 169, 113-127, 2015.

Benitez, F. L., Anderson, L. O., and Formaggio, A. R.: Evaluation of geostatistical techniques to estimate the spatial distribution of aboveground biomass in the Amazon rainforest using high-resolution remote sensing data, Acta Amazonica, 46, 151-160, 2016.

Breiman, L.: Random forests, Machine Learning, 45, 5-32, 2001.

Bustamante, M. M., Roitman, I., Aide, T. M., Alencar, A., Anderson, L., Aragão, L., Asner, G. P., Barlow, J., Berenguer, E., and Chambers, J.: Towards an integrated monitoring framework to assess the effects of tropical forest degradation and recovery on carbon stocks and biodiversity, Global Change Biology, 22, 92-109, 2016.

Chave, J., Réjou-Méchain, M., Búrquez, A., Chidumayo, E., Colgan, M. S., Delitti, W. B. C., Duque, A., Eid, T., Fearnside, P. M., Goodman, R. C., Henry, M., Martínez-Yrízar, A., Mugasha, W. A., Muller-Landau, H. C., Mencuccini, M., Nelson, B. W., Ngomanda, A., Nogueira, E. M., Ortiz-Malavassi, E., Pélissier, R., Ploton, P., Ryan, C. M., Saldarriaga, J. G., and Vieilledent, G.: Improved allometric models to estimate the aboveground biomass of tropical trees, Global Change Biology, 20, 3177-3190, 10.1111/gcb.12629, 2014.

Chen, Q.: Modeling aboveground tree woody biomass using national-scale allometric methods and airborne lidar, ISPRS Journal of Photogrammetry and Remote Sensing, 106, 95-106, 10.1016/j.isprsjprs.2015.05.007, 2015.

Chen, Q., Laurin, G. V., and Valentini, R.: Uncertainty of remotely sensed aboveground biomass over an African tropical forest: Propagating errors from trees to plots to pixels, Remote Sensing of Environment, 160, 134-143, 2015.

Cliff, A., and Ord, V. J.: Spatial processes: model and applications, Pion Ltd, London, 1981.

Conti, G., Gorné, L. D., Zeballos, S. R., Lipoma, M. L., Gatica, G., Kowaljow, E., Whitworth-Hulse, J. I., Cuchietti, A., Poca, M., Pestoni, S., and Fernandes, P. M.: Developing allometric models to predict the individual aboveground biomass of shrubs worldwide, Global Ecology and Biogeography, 28, 961-975, 10.1111/geb.12907, 2019.

Djomo, A. N., Picard, N., Fayolle, A., Henry, M., Ngomanda, A., Ploton, P., McLellan, J., Saborowski, J., Adamou, I., and Lejeune, P.: Tree allometry for estimation of carbon stocks in African tropical forests, Forestry: An International Journal of Forest Research, 89, 446-455, 10.1093/forestry/cpw025, 2016.

Djomo, A. N., and Chimi, C. D.: Tree allometric equations for estimation of above, below and 
total biomass in a tropical moist forest: Case study with application to remote sensing, Forest Ecology and Management, 391, 184-193, https://doi.org/10.1016/j.foreco.2017.02.022, 2017.

Hearst, M. A., Dumais, S. T., Osman, E., Platt, J., \& Scholkopf, B.: Support vector machines, IEEE Intelligent Systems, 13, 18-28, 1998.

Drucker, H., Burges, C. J. C., Kaufman, L., Smola, A., and Vapnik, V.: Support vector regression machines, Proceedings of the 9th International Conference on Neural Information Processing Systems, Denver, Colorado, 1996.

Du, H., Zhou, G., Fan, W., Ge, H., Xu, X., Shi, Y., and Fan, W.: Spatial heterogeneity and carbon contribution of aboveground biomass of moso bamboo by using geostatistical theory, Plant Ecology, 207, 131-139, 2010.

Elanayar, V. T. S., and Shin, Y. C.: Radial basis function neural network for approximation and estimation of nonlinear stochastic dynamic systems, IEEE Transactions on Neural Networks, 5, 594-603, 1994.

Fassnacht, F. E., Hartig, F., Latifi, H., Berger, C., Hernández, J., Corvalán, P., and Koch, B.: Importance of sample size, data type and prediction method for remote sensing-based estimations of aboveground forest biomass, Remote Sensing of Environment, 154, 102-114, 2014.

Fayolle, A., Doucet, J.-L., Gillet, J.-F., Bourland, N., and Lejeune, P.: Tree allometry in Central Africa: Testing the validity of pantropical multi-species allometric equations for estimating biomass and carbon stocks, Forest Ecology and Management, 305, 29-37, https://doi.org/10.1016/j.foreco.2013.05.036, 2013.

Frey, U. J., Klein, M., and Deissenroth, M.: Modelling complex investment decisions in Germany for renewables with different machine learning algorithms, Environmental Modelling \& Software, 118, 61-75, https://doi.org/10.1016/j.envsoft.2019.03.006, 2019.

Gao, L., and Hailu, A.: Ranking management strategies with complex outcomes: An AHP-fuzzy evaluation of recreational fishing using an integrated agent-based model of a coral reef ecosystem, Environmental Modelling \& Software, 31, 3-18, https://doi.org/10.1016/j.envsoft.2011.12.002, 2012.

Gao, L., Bryan, B. A., Nolan, M., Connor, J. D., Song, X., and Zhao, G.: Robust global sensitivity analysis under deep uncertainty via scenario analysis, Environmental modelling \& software, 76, 154-166, 2016.

Gleason, C. J., and Im, J.: Forest biomass estimation from airborne LiDAR data using machine learning approaches, Remote Sensing of Environment, 125, 80-91, 2012.

Görgens, E. B., Montaghi, A., and Rodriguez, L. C. E.: A performance comparison of machine learning methods to estimate the fast-growing forest plantation yield based on laser scanning metrics, Computers and Electronics in Agriculture, 116, 221-227, https://doi.org/10.1016/j.compag.2015.07.004, 2015 .

Hansen, H. E., Gobakken, T., Bollandsås, M. O., Zahabu, E., and Næsset, E.: Modeling 
Aboveground Biomass in Dense Tropical Submontane Rainforest Using Airborne Laser Scanner Data, Remote Sensing, 7, 10.3390/rs70100788, 2015.

He, C., Tian, J., Shi, P., and Hu, D.: Simulation of the spatial stress due to urban expansion on the wetlands in Beijing, China using a GIS-based assessment model, Landscape and Urban Planning, 101, 269-277, https://doi.org/10.1016/j.landurbplan.2011.02.032, 2011.

Houghton, R. A., Hall, F., and Goetz, S. J.: Importance of biomass in the global carbon cycle, Journal of Geophysical Research Biogeosciences, 114, G00E03, 2009.

Hu, M. G., Wang, J. F., Zhao, Y., and Jia, L.: A B-SHADE based best linear unbiased estimation tool for biased samples, Environmental Modelling \& Software, 48, 93-97, 2013.

Huang, H., Liu, C., Wang, X., Zhou, X., and Gong, P.: Integration of multi-resource remotely sensed data and allometric models for forest aboveground biomass estimation in China, Remote Sensing of Environment, 221, 225-234, https://doi.org/10.1016/j.rse.2018.11.017, 2019.

Ioki, K., Tsuyuki, S., Hirata, Y., Phua, M.-H., Wong, W. V. C., Ling, Z.-Y., Saito, H., and Takao, G.: Estimating above-ground biomass of tropical rainforest of different degradation levels in Northern Borneo using airborne LiDAR, Forest Ecology and Management, 328, 335-341, https://doi.org/10.1016/j.foreco.2014.06.003, 2014.

Jachowski, N. R. A., Quak, M. S. Y., Friess, D. A., Duangnamon, D., Webb, E. L., and Ziegler, A. D.: Mangrove biomass estimation in Southwest Thailand using machine learning, Applied Geography, 45, 311-321, https://doi.org/10.1016/j.apgeog.2013.09.024, 2013.

Kim, E., Lee, W.-K., Yoon, M., Lee, J.-Y., Son, Y., and Abu Salim, K.: Estimation of Voxel-Based Above-Ground Biomass Using Airborne LiDAR Data in an Intact Tropical Rain Forest, Brunei, Forests, 7, 10.3390/f7110259, 2016.

Latifi, H., Nothdurft, A., and Koch, B.: Non-parametric prediction and mapping of standing timber volume and biomass in a temperate forest: application of multiple optical/LiDAR-derived predictors, Forestry: An International Journal of Forest Research, 83, 395-407, 10.1093/forestry/cpq022, 2010.

Li, J., Heap, A. D., Potter, A., and Daniell, J. J.: Application of machine learning methods to spatial interpolation of environmental variables, Environmental Modelling \& Software, 26, 1647-1659, https://doi.org/10.1016/j.envsoft.2011.07.004, 2011.

Lu, Z., Lin, F., and Ying, H.: DESIGN OF DECISION TREE VIA KERNELIZED HIERARCHICAL CLUSTERING FOR MULTICLASS SUPPORT VECTOR MACHINES, Cybernetics and Systems, 38, 187-202, 10.1080/01969720601139058, 2007.

Malhi, Y., Phillips, O. L., Chave, J., Condit, R., Aguilar, S., Hernandez, A., Lao, S., and Perez, R.: Error propagation and scaling for tropical forest biomass estimates, Philosophical Transactions of the Royal Society of London. Series B: Biological Sciences, 359, 409-420, 10.1098/rstb.2003.1425, 2004.

Marvin, D. C., Asner, G. P., Knapp, D. E., Anderson, C. B., Martin, R. E., Sinca, F., and Tupayachi, R.: Amazonian landscapes and the bias in field studies of forest structure and biomass, Proceedings of the National Academy of Sciences of the United States of America, 111, 5224-5232, 2014. 
McRoberts, R. E., Chen, Q., Domke, G. M., Ståhl, G., Saarela, S., and Westfall, J. A.: Hybrid estimators for mean aboveground carbon per unit area, Forest Ecology and Management, 378, 44-56, 10.1016/j.foreco.2016.07.007, 2016.

McRoberts, R. E., Chen, Q., Gormanson, D. D., and Walters, B. F.: The shelf-life of airborne laser scanning data for enhancing forest inventory inferences, Remote Sensing of Environment, 206, 254-259, 10.1016/j.rse.2017.12.017, 2018.

Mendoza-Ponce, A., and Galicia, L.: Aboveground and belowground biomass and carbon pools in highland temperate forest landscape in Central Mexico, Forestry: An International Journal of Forest Research, 83, 497-506, 10.1093/forestry/cpq032, 2010.

Morel, A. C., Fisher, J. B., and Malhi, Y.: Evaluating the potential to monitor aboveground biomass in forest and oil palm in Sabah, Malaysia, for 2000-2008 with Landsat ETM+ and ALOS-PALSAR, International Journal of Remote Sensing, 33, 3614-3639, 2012.

Paul, K. I., Roxburgh, S. H., Chave, J., England, J. R., Zerihun, A., Specht, A., Lewis, T., Bennett, L. T., Baker, T. G., Adams, M. A., Huxtable, D., Montagu, K. D., Falster, D. S., Feller, M., Sochacki, S., Ritson, P., Bastin, G., Bartle, J., Wildy, D., Hobbs, T., Larmour, J., Waterworth, R., Stewart, H. T., Jonson, J., Forrester, D. I., Applegate, G., Mendham, D., Bradford, M., O'Grady, A., Green, D., Sudmeyer, R., Rance, S. J., Turner, J., Barton, C., Wenk, E. H., Grove, T., Attiwill, P. M., Pinkard, E., Butler, D., Brooksbank, K., Spencer, B., Snowdon, P., O'Brien, N., Battaglia, M., Cameron, D. M., Hamilton, S., McAuthur, G., and Sinclair, J.: Testing the generality of above-ground biomass allometry across plant functional types at the continent scale, Global Chang Biology, 22, 2106-2124, 10.1111/gcb.13201, 2016.

Picard, N., Rutishauser, E., Ploton, P., Ngomanda, A., and Henry, M.: Should tree biomass allometry be restricted to power models?, Forest Ecology and Management, 353, 156-163, https://doi.org/10.1016/j.foreco.2015.05.035, 2015.

Povak, N. A., Hessburg, P. F., McDonnell, T. C., Reynolds, K. M., Sullivan, T. J., Salter, R. B., and Cosby, B. J.: Machine learning and linear regression models to predict catchment-level base cation weathering rates across the southern Appalachian Mountain region, USA, Water Resources Research, 50, 2798-2814, 10.1002/2013WR014203, 2014.

Propastin, P.: Modifying geographically weighted regression for estimating aboveground biomass in tropical rainforests by multispectral remote sensing data, International Journal of Applied Earth Observation and Geoinformation, 18, 82-90, 2012.

Qiu, Q., Yun, G., Zuo, S., Yan, J., Hua, L., Ren, Y., Tang, J., Li, Y., and Chen, Q.: Variations in the biomass of Eucalyptus plantations at a regional scale in Southern China, Journal of Forestry Research, 29, 1263-1276, 10.1007/s11676-017-0534-0, 2018.

Raczko, E., and Zagajewski, B.: Comparison of support vector machine, random forest and neural network classifiers for tree species classification on airborne hyperspectral APEX images, European Journal of Remote Sensing, 50, 144-154, 10.1080/22797254.2017.1299557, 2017.

Rangel, T. F., and Bini, L. M.: SAM: A comprehensive application for Spatial Analysis in 
Macroecology, Ecography, 33, 46-50, 2010.

Ren, Y., Zhang, C., Zuo, S., and Li, Z.: Scaling up of biomass simulation for Eucalyptus plantations based on landsenses ecology, International Journal of Sustainable Development \& World Ecology, 24, 135-148, 2017.

Rosenberg, M. S., and Anderson, C. D.: PASSaGE: Pattern Analysis, Spatial Statistics and Geographic Exegesis. Version 2, Methods in Ecology and Evolution, 2, 229-232, 2011.

Saatchi, S. S., Harris, N. L., Brown, S., Lefsky, M., Mitchard, E. T. A., Salas, W., Zutta, B. R., Buermann, W., Lewis, S. L., Hagen, S., Petrova, S., White, L., Silman, M., and Morel, A.: Benchmark map of forest carbon stocks in tropical regions across three continents, Proceedings of the National Academy of Sciences of the United States of America, 108, 9899-9904, 10.1073/pnas.1019576108, 2011.

Schabenberger, O., and Gotway, C. A.: Statistical methods for spatial data analysis, Chapman \& HalloCRC, Boca Raton, 2005.

Stojanova, D., Panov, P., Gjorgjioski, V., Kobler, A., and Džeroski, S.: Estimating vegetation height and canopy cover from remotely sensed data with machine learning, Ecological Informatics, 5, 256-266, https://doi.org/10.1016/j.ecoinf.2010.03.004, 2010.

Van der Laan, C., Verweij, P. A., Quiñones, M. J., and Faaij, A. P.: Analysis of biophysical and anthropogenic variables and their relation to the regional spatial variation of aboveground biomass illustrated for North and East Kalimantan, Borneo, Carbon Balance and Management, 9, 8, 2014.

Viana, H., Aranha, J., Lopes, D., and Cohen, W. B.: Estimation of crown biomass of Pinus pinaster stands and shrubland above-ground biomass using forest inventory data, remotely sensed imagery and spatial prediction models, Ecological Modelling, 226, 22-35, 2012.

Wang, J.-F., Zhang, T.-L., and Fu, B.-J.: A measure of spatial stratified heterogeneity, Ecological Indicators, 67, 250-256, https://doi.org/10.1016/j.ecolind.2016.02.052, 2016.

Wang, J. F., Li, X. H., Christakos, G., Liao, Y. L., Zhang, T., Gu, X., and Zheng, X. Y.: Geographical Detectors-Based Health Risk Assessment and its Application in the Neural Tube Defects Study of the Heshun Region, China, International Journal of Geographical Information Science, 24, 107-127, 2010.

Xia, C. L., Xiu, J.: RBF ANN Nonlinear Prediction Model Based Adaptive PID Control of Switched Reluctance Motor, Proceedings of the CSEE, 27, 626-635, 10.1007/11893295_69, 2007.

Xu, C. D., Wang, J. F., Hu, M. G., and Li, Q. X.: Interpolation of Missing Temperature Data at Meteorological Stations Using P-BSHADE*, Journal of Climate, 26, 7452-7463, 2013.

Zhang, J., Huang, S., Hogg, E. H., Lieffers, V., Qin, Y., and He, F.: Estimating spatial variation in Alberta forest biomass from a combination of forest inventory and remote sensing data, Biogeosciences, 11, 2793-2808, 10.5194/bg-11-2793-2014, 2014.

Zheng, D., Rademacher, J., Chen, J., Crow, T., Bresee, M., Moine, J. L., and Ryu, S. R.: 
https://doi.org/10.5194/bg-2020-36

Preprint. Discussion started: 25 February 2020

(C) Author(s) 2020. CC BY 4.0 License.

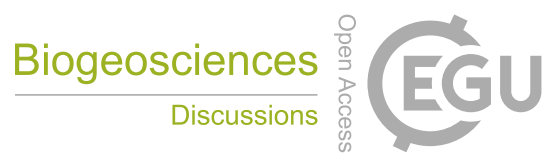

(c) (1)

813 Estimating aboveground biomass using Landsat 7 ETM+ data across a managed landscape in northern

814 Wisconsin, USA, Remote Sensing of Environment, 93, 402-411, 2004. 